\title{
Title: Oxytocin is associated with infant-care behavior and motivation in cooperatively breeding marmoset
}

\section{monkeys}

Authors: Christa Finkenwirth ${ }^{1}$, Eloisa Martins ${ }^{1}$, Tobias Deschner ${ }^{2}$ and Judith M. Burkart ${ }^{1}$

\author{
Affiliations: \\ ${ }^{1}$ Anthropological Institute and Museum, University of Zurich, Winterthurerstrasse 190, 8057 \\ Zurich, Switzerland \\ ${ }^{2}$ Max-Planck Institute for Evolutionary Anthropology, Deutscher Platz 6, 04103 Leipzig, \\ Germany
}

Correspondence and requests should be addressed to:

Christa Finkenwirth

Anthropological Institute and Museum

Winterthurerstr. 190

CH-8057 Zurich

Tel. +4144635 5448

Email: christa.finkenwirth@uzh.ch 


\section{Abstract}

The neurohormone oxytocin (OT) is positively involved in the regulation of parenting and social bonding in mammals, and may thus also be important for the mediation of alloparental care. In cooperatively breeding marmosets, infants are raised in teamwork by parents and adult and subadult non-reproductive helpers (usually older siblings). Despite high intrinsic motivation, which may be mediated by hormonal priming, not all individuals are always equally able to contribute to infant-care due to competition among care-takers. Among the various care-taking behaviors, proactive food sharing may reflect motivational levels best, since it can be performed ad libitum by several individuals even if competition among surplus care-takers constrains access to infants. Our aim was to study the link between urinary OT levels and care-taking behaviors in groupliving marmosets, while taking affiliation with other adults and infant age into account. Over eight reproductive cycles, 26 individuals were monitored for urinary baseline OT, care-taking behaviors (baby-licking, -grooming, -carrying, and proactive food sharing), and adult-directed affiliation. Mean OT levels were generally highest in female breeders and OT increased significantly in all individuals after birth. During early infancy, high urinary OT levels were associated with increased infant-licking but low levels of adult-affiliation, and during late infancy, with increased proactive food sharing. Our results show that, in marmoset parents and alloparents, OT is positively involved in the regulation of care-taking, thereby reflecting the changing needs during infant development. This particularly included behaviors that are more likely to reflect intrinsic care motivation, suggesting a positive link between OT and motivational regulation of infant-care. 
Key words: oxytocin, hormonal priming, alloparental care, proactive food sharing, motivation, cooperative breeding, Callithrix jacchus 


\section{Introduction}

Over the last decades, oxytocin (OT) has been shown to be versatilely involved in the regulation of mammalian social behaviors and bonding (Lim and Young, 2006), most importantly in maternal care and mother-infant bonds (Kendrick, 2000; Uvnäs-Moberg, 1996). Its ancient functions in female reproduction, related to cervix and uterus distension during labor (Blanks and Thornton, 2003; Kendrick and Keverne, 1989; Landgraf et al., 1983; Nissen et al., 1995), milk injection during lactation (Uvnäs-Moberg et al., 2001), and the regulation of maternal behavior (Feldman, 2007; Kendrick, 2000; Kendrick et al., 1987; Pedersen et al., 1994; Uvnäs-Moberg, 1996), probably formed the basis for derived OT functions in non-maternal social contexts (Broad et al., 2006; Kendrick, 2000; Lim and Young, 2006). However, less is known about the role of OT in alloparental care, i.e. care provided by non-mothers, including paternal care provided by fathers.

Among nonhuman primates, alloparental care is most frequent in the cooperatively breeding callitrichid monkeys (Digby et al., 2007). Whereas all group members cooperate in rearing offspring, reproduction is usually restricted to a dominant breeding pair (Digby et al., 2007; Garber, 1997; Goldizen, 2003), but mating systems and group composition show considerable flexibility, in particular in wild callitrichids (Garber et al., 2015; Goldizen, 1988; Solomon and French, 1997; Sussman and Garber, 1987). Reproductive success depends on the availability of alloparental care, which increases infant growth and survival, especially in common marmosets (Koenig, 1995; Rothe et al., 1993) but also in other callitrichid species (Sussman and Garber, 1987). After the first post-partum days, during which mothers typically are the primary care-takers in common marmosets (Koenig and Rothe, 1991; Mills et al., 2004), 
help is provided by fathers and other group members, who are mostly, but not exclusively, the adult and sub-adult offspring of the breeding pair, and who delay dispersal and help rearing their siblings (Digby et al., 2007; Garber, 1997; Goldizen, 1987; Yamamoto et al., 2014).

During early infancy, helping behavior mostly consists of carrying the offspring, which bears high energetic costs to carriers (Schradin and Anzenberger, 2001). Nevertheless, caretakers also compete over access to infants (Mills et al., 2004; Yamamoto and Box, 1997; Zahed et al., 2010), and particularly female helpers are not always tolerated by other group members to handle and carry infants as much as they attempt to (Albuquerque, 1999; Price, 1991). Hence, individual infant-carrying contributions may not necessarily correspond to intrinsic motivational levels, especially if more care-takers than infants are available. During later infancy until juvenile age, carrying continually decreases and is complemented by food provisioning to immatures. In contrast to infant-carrying, food provisioning can always be performed ad libitum and is usually not constrained by other group members. During peak provisioning periods, adults share up to $63 \%$ of provided food items with immatures, and $40 \%$ of these food transfers occur proactively (Martins et al., in preparation), i.e. are initiated by the adult who offers the food to the immature (Brown et al. 2004). Non-reproductive helpers show particularly high levels of proactive food sharing (48\%) during that time, Alloparents thus seem to have high intrinsic caretaking motivation, and, in fact, helping in subordinates is not enforced by breeders through coercion or punishment (Snowdon and Cronin, 2007).

In marmoset mothers, labor and lactation as well as maternal infant-care after birth are accompanied by a number of hormonal changes: Towards labor, estradiol and progesterone levels decrease drastically (Chambers and Hearn, 1979) and are linked to post-partum sexual cycling independently of lactation (Kholkute, 1984), whereas prolactin levels increase and 
remain elevated during lactation (McNeilly et al., 1981). Reproduction-related OT patterns in callitrichid monkeys may be comparable to those of other primates, where plasma OT is elevated during late pregnancy and parturition (Hirst and Thorburn, 1996; Novy and Haluska, 1994), and during lactation in rhesus monkeys (Macaca mulatto, Amico et al., 1990) but not in Cynomolgus monkeys (Macaca fascicularis, Morris et al., 1980).

The hormones mentioned above are also involved in maternal care-taking behaviors and motivation: Sex steroids positively affect maternal responsiveness during late pregnancy in marmosets and tamarins (Pryce et al., 1993). Likewise, pre-partum estrogen levels are positively linked to subsequent maternal care-taking behavior in tamarins (Pryce et al., 1988), macaques (Bardi et al., 2003), and humans (Fleming et al., 1987; see also Maestripieri, 2001a, 2001b, and Saltzman and Maestripieri, 2011). Conflicting results were described for common marmosets (Pryce et al., 1995), black-tufted marmosets (Fite and French, 2000), gorillas (Bahr et al., 2001), and baboons (Bardi et al., 2004; see also Maestripieri, 2001a; Saltzman and Maestripieri, 2011). Primate maternal behavior can also be influenced by stress-related hormones and neuropeptides (Saltzman and Maestripieri, 2011), whereas there is no indication for a positive link of prolactin and maternal care based on the limited available evidence in marmosets (Saltzman and Abbott, 2005). OT seems to be fundamentally involved in motivational priming during mother-infant bonding in mammals (Carter, 1998; Maestripieri, 2001b) and it is positively involved in maternal behavior in rats (Fahrbach et al., 1984; Pedersen et al., 1994) and sheep (Kendrick et al., 1987), but such effects have not been studied in primates yet.

The neuroendocrine hypothesis suggests that hormonal priming may generally play a vital role in the mediation of infant-care motivation, which may provide an important complement to parenting experience, thus being particularly valuable for inexperienced mothers 
but also alloparental care-takers (Pryce, 1993). However, evidence for such effects in primates is still very limited and partly contradictory. Positive evidence related to non-maternal care exists for example for tamarin fathers, who show changes of testosterone, estradiol, and glucocorticoid levels in the pre-partum period as well as during infant-care, possibly preparing males for the impending birth and parenting role (Ziegler and Snowdon, 2000; Ziegler et al., 2004; Ziegler et al., 1996). Prolactin is also positively linked to infant-care in marmoset fathers and helpers (Dixson and George, 1982; Mota et al., 2006; Mota and Sousa, 2000; Roberts et al., 2001, Schradin and Anzenberger, 2004; Schradin et al., 2003), but these studies suggest that prolactin increases in response to infant-contact and -carrying rather than promoting such behaviors based on motivation-stimulating effects. Accordingly, experienced marmoset fathers could express normal paternal care even if prolactin was suppressed (Almond et al., 2006). In contrast, OT has been shown to stimulate alloparental infant-care motivation in different mammal species: In rodents, peripheral OT administration facilitated alloparental pup-retrieval, -licking, and grooming in females (Bales et al., 2007), and OT-antagonist inhibited such behaviors in males (Bales et al., 2004). Peripheral OT administration also enhanced guarding, feeding, and association with pups in meerkats (Madden and Clutton-Brock, 2011), and intracerebroventricular OT administration facilitated paternal food sharing in marmosets (Saito and Nakamura, 2011). However, it remains to be established whether OT is also positively linked to alloparental behavior and motivation in non-reproductive primate helpers, and whether this link is reflected in endogenous OT responses, since the existing evidence mainly focusses on exogenous OT effects.

The aim of this study was to assess the role of OT in care-taking behavior in family groups of cooperatively breeding marmosets, with particular focus on the relationship between 
OT and care motivation in non-mothers. We longitudinally followed five family groups of common marmosets over a total of eight reproductive cycles. During each reproductive cycle, we collected baseline OT levels from morning void urine and data on care-taking behaviors (infantcarrying, -licking, -grooming, and proactive food sharing) and affiliative behaviors between adult group members (grooming and huddling), over a total period of 12 weeks (starting one week before birth).

First, we investigated whether urinary OT levels increased immediately after infant-birth in mothers and other adult care-takers, which would be consistent with the neuroendocrine priming hypothesis (Pryce, 1993). Based on OT functions in mammalian birth (Kendrick, 2000; Landgraf et al., 1983; Nissen et al., 1995) and lactation (Uvnäs-Moberg et al., 2001), we expected OT levels of breeding females to be elevated after birth and during lactation until infant-weaning. Higher OT levels in response to the presence of new-born offspring were also expected in marmoset fathers, based on findings of positive peripheral OT responses in human parents (Feldman et al., 2011; Gordon et al., 2010; Nissen et al., 1995). Since non-reproductive helpers in marmosets also show high levels of alloparental care (Digby et al., 2007; Goldizen, 1987), we predicted a positive OT response also in helper individuals of both sexes.

Second we analyzed to what extent urinary OT patterns reflect care-taking behaviors, while taking affiliation with other adults, infant age, and care-taking experience into account. We also compared the effects in early and late infancy, because frequencies and importance of the different infant-directed behaviors change over time. The facilitating effects of exogenous OT on paternal food transfer in marmosets (Saito and Nakamura, 2011) and alloparental behaviors in other mammals (Bales et al., 2007; Madden and Clutton-Brock, 2011) suggest that OT may be positively associated with intrinsic care-taking motivation. We therefor expected urinary OT 
levels to be positively linked in particular to those care-taking behaviors that can be freely expressed by all individuals motivated to do so. During early infancy, this arguably mainly includes infant-licking and -grooming, since the immobile infants cannot be carried simultaneously by all motivated care-takers, but infants can be licked and groomed also on the backs of other care-takers. Furthermore, compared to licking and grooming, carrying is a more passive behavior once the infant sits on the carrier and may thus be less related to OT. During late infancy, carrying and licking decrease continuously and food sharing becomes more important. In particular proactive food sharing (Brown et al. 2004), which is initiated by the food possessor rather than a begging immature, was expected to be positively linked to OT during this period because it is dependent on intrinsic proactive motivation (Jaeggi and Gurven, 2013) and can be performed ad libitum by all motivated care-takers.

\section{Methods}

\section{Study animals}

We studied five marmoset family groups (26 individuals from) over a total of eight reproductive cycles. Two groups (ten individuals) were sampled repeatedly over different reproductive cycles during this study. Reproductive cycle order, group identity, size, and composition, as well as individual identity, sex, status (breeder or helper), age, and care-taking experience (number of raised offspring for breeders and the number of raised siblings for helpers) are listed in supplementary table S1. Juvenile group members from previous reproductive cycles, who were younger than 10 months and did not yet participate in alloparenting of new born offspring, were not part of this study and are not listed in table S1. 
One sub-adult individual (Nikita) was actively participating in alloparenting in reproductive cycle 8 and was hence included in the analysis with the other (adult) care-takers. All groups were housed in standardized enclosures (depending on group size, one or multiple basic cage units; each measuring $2.4 \mathrm{~m}$ height $\times 1.5 \mathrm{~m}$ depth $\times 0.8 \mathrm{~m}$ width). All enclosures were connected to spacious outdoor areas and equipped with a sleeping box, a water dispenser, several wooden

climbing structures, an infrared lamp and a mulch floor. The animals were housed under natural light with additional artificial light on a $12 \mathrm{~h} / 12 \mathrm{~h}$ light-dark cycle and UV light (300W). Their diet consisted of a vitamin and calcium-enriched porridge in the morning, fresh fruits and vegetables over midday, as well as gum and mealworms in the afternoon, and water was available ad libitum. A special cage unit that was only accessible in the morning during urine collection (Anzenberger and Gossweiler, 1993) was attached to each home cage. On urine sampling days, mealworms were fed in the urine cages in the morning instead of afternoon.

\section{Urine sampling and $O T$ detection}

Baseline OT levels were detected from individual morning urine samples that were collected during each reproductive cycle. For each adult group member, urine was collected two times per week over a period of six to 12 consecutive weeks, starting one week before birth. The sampling was restricted to morning-void urine, in order to measure hormonal baseline values and to minimize confounding effects of circadian rhythms. Urine was collected non-invasively either in a urine cage or with a plastic cup in the home cage, as described elsewhere (Finkenwirth et al., 2015). Urine samples were labelled right after collection and stored immediately at $-20^{\circ} \mathrm{C}$ in $1 \mathrm{ml}$ portions until analysis. 
OT was measured in the endocrinology laboratory of the department of Primatology at the Max Planck Institute of Evolutionary Anthropology, Leipzig, Germany, using commercial OT assay kits (Assay Designs, Cat no. ADI-900-153A-0001). The procedure was developed and validated by Seltzer and Ziegler (2007) and Snowdon et al. (2010), and slightly adapted for our purpose, as previously described in Finkenwirth et al. (2015). A detailed protocol is provided in supplementary file S2. Because a new antibody component was developed by Enzo Assay Designs and provided with the OT assay kit during our study, OT measurements of reproductive cycle 1-3 and 4-8 were based on different OT assays kits with slightly different binding capacities (assay A and B). OT concentrations measured with assay B were approximately two times higher than with assay A. We therefore measured 33 samples with both kits, and found OT concentrations to be highly correlated between the two assays (Pearson correlation: $\mathrm{N}=33, \mathrm{r}=$ $0.82, \mathrm{p}<0.001$ ), and quality criteria of both assays were very similar. Mean intra-assay coefficients of variation for high $(250 \mathrm{pg} / \mathrm{ml})$ and low $(50 \mathrm{pg} / \mathrm{ml})$ OT standards were $9.59 \%$ and $13.76 \%$ for assay A, and $6.51 \%$ and $14.36 \%$ for assay B, respectively. Mean inter-assay variation coefficients of variation for high and low OT standards were $12.48 \%$ and $19.26 \%$ for assay A, and $8.23 \%$ and $13.56 \%$ for assay B, respectively. Prior to statistical analysis, all OT concentrations were corrected for creatinine levels to control for variable urine concentration and ln-transformed to reach normal distribution. In order to analyze OT levels across all reproductive cycles despite the assay-dependent variation in OT measurements, ln-transformed OT concentrations were also z-standardized within each assay ( $\mathrm{Z} \ln \mathrm{pg} \mathrm{OT} / \mathrm{mg} \mathrm{Crt}$ ). Information on original OT measurements ( $\mathrm{pg} / \mathrm{mg} \mathrm{Crt})$ is provided in supplementary table $\mathrm{S} 1 \mathrm{in}$ form of individual mean \pm SD OT levels. 


\section{Behavioral data collection}

Along each reproductive cycle, we collected data on care-taking behaviors, adultaffiliation, and proactive food sharing rates. A detailed sampling protocol of behavioral and hormonal data along the eight reproductive cycles is provided in supplementary table S3. Caretaking behaviors included infant-carrying, which was recorded daily (between 8 am and $5 \mathrm{pm}$ ) in hourly group scans during 100 days after birth, and infant-licking and -grooming, which was recorded two to three times per week as all occurrences in continuous group observations of one hour (data available for birth 4-8). Adult-directed affiliative behaviors included grooming (picking the fur or skin of a partner with hands or mouth) and huddling (resting in direct body contact), which was recorded as all occurrences along with the infant-directed behavior during the continuous group observations. Observation sessions were equally distributed between morning and afternoon (9-12 am or 1-5 pm). For analysis, adult-grooming and -huddling were combined into one variable (data available for all groups). All observed behaviors and carrying scans were corrected for observation time or the number of scans, respectively, and carrying is expressed as percentage of the total number of scans $(100 *$ scans carried/total scans). Finally, all affiliation variables (infant-licking and -grooming and adult-affiliation) were ln-transformed in order to reach normal distributions prior to statistical analysis.

Proactive food sharing was characterized by i) the donor keeping the food in the mouth or hand while emitting food calls and waiting for a recipient and ii) by the absence of begging from recipients (Brown et al., 2004). Proactive food sharing was assessed experimentally two to three times per week (between 9-12 am or 1-5 pm) for each adult care-taker in a group. To do so, a specific donor received three to five preferred food items (small crickets) one after another, and we recorded the frequencies and characteristics of sharing (direction, vocalizations, begging 
intensity, pro- and reactivity, see also Martins et al., in preparation). After receiving the food item, donors usually first moved into distance of the group and then either started to eat the food themselves or to initiate proactive food sharing with food calls. If donors were harassed by potential recipients prior to sharing or if they shared food only after begging by recipients, we recorded this behavior as resisted or reactive sharing, which was not included in the present analysis. To avoid saturation of infants and keep their interest in food items high, we tested only up to three adult care-takers per group and day. Proactive food sharing rates were expressed as percentage of the total number of food items received $(100 * \mathrm{~N}$ food items shared proactively/N food items received).

\section{Statistics}

In order to investigate the relationship between urinary OT levels and infant birth, caretaking behaviors, adult-affiliation, and infant age, we performed several linear mixed-effect models with restricted maximum likelihood estimation ( $\mathrm{R}$ version 3.1.3, lme package). All models included the random factors of individual nested in family group, and reproductive cycle nested in family group, to control for the repeated occurrence of the same individuals across different reproductive cycles (relevant for individuals in the groups 'Nina' and 'Lancia', see supplementary table S1).

First, we tested whether OT increased after infant-birth (model 1), by comparing data from the late pre-partum period (one week before birth) with the early post-partum period (weeks zero and one). Sampling period (pre- and post-birth), status (breeder and helper), and sex (female and male) were included as fixed factors, and the model was based on individual mean values 
from all 26 individuals of the eight reproductive cycles $(\mathrm{N}=70$ samples, missing values from week -1 of two groups).

Second, we investigated longitudinal changes in urinary OT levels and care-taking behaviors (infant-carrying, -licking, -grooming, and proactive food sharing) and the link between both over 11 weeks after birth. We first compared overall changes in care-taking behaviors between early (week 0-5) and late infancy (week 6-11) based on individual mean values for each time block. To do so, we performed four linear mixed models including infant-carrying (model 2), -licking (model 3), -grooming (model 4), or proactive food sharing (model 5) as dependent variable, sex, status, and infant age as fixed factors (supplementary tables S4). Infant age was a block variable with two levels (block $1=$ week $0-5$, block $2=$ week $6-11$ post-partum). For infant-carrying, these changes were also analyzed separately for status-sex groups, using paired samples t-test and Cohen's d for effect size estimation. This was not possible for infant-licking and -grooming due to limited sample size.

Third, we tested the link between weekly mean OT values, care-taking behaviors (infantcarrying, -licking, -grooming, and proactive food sharing), and adult-affiliation, based on the data from reproductive cycles 4-8 ( $\mathrm{N}=236$ samples from 22 individuals; see supplementary table S3). To do so, we first performed a full model (model 6) including all behavioral variables, status, sex, infant age, and individual care-taking experience as fixed factors (supplementary table S4). Care-taking experience was the number of raised offspring for breeders and the number of raised siblings for helpers. We also included the interaction between status and sex, since effects in female breeders, male breeders, female helpers, and male helpers may differ. Second, in order to evaluate the changing impact of behavioral variables on OT during infant development, we performed separate analyses for early (model 7) and late infancy (model 8). We 
tested different models that included all factors of the full model, as well as biologically

meaningful interactions. The best-fitting model was selected based on the Akaike information criterion (AIC) estimation. In all models, approximate normality and homogeneity of residuals was assessed by visual inspection of residuals plotted against fitted values and a qq-plot. All model assumptions were met.

\section{Results}

\section{Comparing pre- and post-birth OT levels}

In breeders as well as helpers, urinary OT levels increased within the first days after the birth of new infants (figure 1). This increase was highly significant $(\mathrm{t}=4.98, \mathrm{p}<0.0001$, model 1 , table 1). The model also showed strong effects of status and sex as well as an interaction of status*sex, as the highest OT levels were present in breeder females (figure 1).

\section{OT and care-taking behaviors}

Over the total study period of 12 weeks, starting one week prior to birth, mean urinary OT levels in breeder females, breeder males, helper females, and helper males underwent substantial longitudinal changes (figure 2, data from all 8 reproductive cycles). In all classes of animals, a significant OT peak occurred in the early post-partum period (see also table 1, model 1). Breeder females generally showed the highest OT levels (tables $1 \& 2$, models $1 \& 7$ ) and their post-partum OT remained on a high plateau that only declined towards week five, when infants start to eat solid food regularly and lactation decreases (indicated by the arrow in figure 2). 
Longitudinal changes of care-taking behaviors including infant-carrying, -licking, grooming, and proactive food sharing over 11 weeks after birth are shown in figure 3A-D. Mean rates of carrying, licking and grooming were highest during early infancy compared to late infancy (carrying: $\mathrm{t}=-5.29, \mathrm{p}<0.001$, model 2; licking: $\mathrm{t}=-6.83, \mathrm{p}<0.001$, model 3; grooming: $\mathrm{t}=-2.25, \mathrm{p}=0.030$, model 4 ; supplementary table S4), following a decreasing trend. Infantcarrying rates of breeders and helpers showed compensating patterns over time: Whereas carrying decreased from early to late infancy in breeder females $(\mathrm{t}(6)=9.03, \mathrm{p}<0.001, \mathrm{~d}=$ 2.85), breeder males $(\mathrm{t}(7)=5.73, \mathrm{p}=0.001, \mathrm{~d}=2.15)$, and helper males $(\mathrm{t}(11)=2.89, \mathrm{p}=0.015$, $\mathrm{d}=1.26$ ), it showed a strong increasing trend in female helpers, who only reached peak levels of carrying in week seven post-partum $(\mathrm{t}(12)=-1.77, \mathrm{p}=0.10, \mathrm{~d}=-0.51)$. Mean proactive food sharing increased significantly to peak levels after week five post-partum (during late infancy) in all individuals $(\mathrm{t}=9.52, \mathrm{p}<0.0001$, model 5 ; supplementary table $\mathrm{S} 4)$, which was congruent with the transition of the infants' diet from milk to solid food and with increasing infant mobility. Particularly high levels of proactive food sharing were reached in female and male helpers as well as male breeders between week seven and nine, and this time was also characterized by a new increasing trend in urinary OT levels in breeders as well as helpers (figure 2).

The relationship between individual urinary OT levels, care-taking behaviors, and adultaffiliation over the total study period of 11 weeks after birth was first assessed in a full linear mixed model (model 6, supplementary table S4). OT was significantly higher in breeders than helpers and in females than males. The interaction effect status*sex reflects the higher OT levels in female breeders compared to other individuals (figures 1 and 2). OT decreased from early towards late infancy, as indicated by the negative effect of infant age. Among the care-taking behaviors, proactive food sharing had a positive effect on OT, but no effect was found for infant- 
carrying, -licking, and -grooming. Negative effects emanated from adult-affiliation and individual parenting experience (number of raised offspring or siblings).

To evaluate the changing impact of care-taking behaviors and adult-affiliation on OT during infant development, we analyzed the data separately for early and late infancy. During early infancy, we found a strongly positive effect of infant-licking, and higher OT levels in females compared to males and in breeders compared to helpers (table 2, model 7). Affiliation with adults had a negative effect. During late infancy, breeders still had higher OT levels than helpers, but the effects of infant-licking, sex, and adult-affiliation effects vanished. Instead, proactive food sharing was positively linked to OT levels during this period (table 2, model 8).

\section{Discussion}

This study systematically investigated longitudinal changes of urinary OT related to caretaking behaviors over three months after birth in group-living marmoset breeders and alloparental helpers. Specifically, we tested whether OT increased in breeders and helpers directly after infant birth, and to what extent changes in OT reflected the changing needs during infant development and care-taking motivation.

First, we found that in all marmoset care-takers, OT levels increased immediately after the birth of new offspring. OT levels in the pre-partum week and early post-partum period were highest in female breeders, consistent with the general role of OT in parturition (Blanks and Thornton, 2003) and lactation (Uvnäs-Moberg et al., 2001). However, the post-partum OT increase in fathers and non-reproductive helpers suggests that the role of OT extends beyond maternal reproduction and is also linked to infant-care, in both parents and alloparents. This is 
consistent with the neuroendocrine hypothesis that emphasizes the relevance of hormonal priming mechanisms underlying infant-care motivation (Pryce, 1993).

Second, we found that during early infancy, longitudinal changes of post-partum OT levels were positively linked to infant-licking, and during late infancy, to proactive food sharing, in both breeders and helpers. Furthermore, during early infancy - the most intense time of infantcare - adult-affiliation was negatively correlated with urinary OT levels, arguably reflecting time-budget trade-offs between infant-care and affiliation with other adults.

The diverging patterns of OT effects in early and late infancy correspond to the changing developmental needs of the infants: licking occurs mainly with young infants and food sharing with older infants. In early infancy, infant-carrying and -grooming are frequent too, but they are not related to OT. Infant-licking, more than -grooming or -carrying, arguably involves highly intense chemical stimulation, which might be responsible for its strong link with OT. Physical contact and effort during infant-grooming or -carrying, however, did not exert any effect on OT. Together with the strong effect of proactive food sharing on OT, this indicates that OT levels during periods of intense infant-care do not simply result from physical contact, as suggested for prolactin (Mota et al., 2006). Rather, OT may be involved in the motivational regulation of infant-care, which is also consistent with stimulating effects of exogenous OT-administration on maternal (Kendrick et al., 1987; Pedersen et al., 1994) and non-maternal (Bales et al., 2007; Madden and Clutton-Brock, 2011; Saito and Nakamura, 2011) care-taking in mammals. Nonetheless, the causal interpretation of effect directions in our findings is limited due to the non-invasive study design. Finally, potential motivation stimulating OT effects and OT responses to chemical or physical stimuli may also be intertwined bi-directionally, especially if interactions with other hormones are involved. 
The hypothesis that OT may be positively associated with care-taking motivation is particularly supported by the positive link of OT and proactive food sharing during late infancy. Proactive food sharing, the only correlate of OT in late infancy, is a care-taking behavior that can be performed almost without constraint, and is thus thought to be a direct indicator of an intrinsic motivation to care (note that food sharing was assessed experimentally, which also controlled for the possibility of infant-satiation). However, infant-licking during early infancy may also be a good proxy for care-motivation - more so than carrying or grooming. Carrying was not possible for all motivated care-takers at the same time because a maximum of two infants per group were available. Furthermore, infant-carrying rates may also be influenced and biased by other social constraints, e.g. female breeders could be protective and restrict the access to infants (mostly towards female helpers), arguably to minimize the risk of infanticide (Albuquerque, 1999; Digby, 1995; Yamamoto et al., 2010; Yamamoto et al., 2009). Finally, older and more mobile infants play an increasingly active role in choosing their carriers. Grooming levels may be a weak proxy for care motivation because they may not exclusively represent infant-directed grooming, since infants were often groomed on the back of other adult carriers, and adultdirected grooming could not always be clearly excluded in the observation.

During the first post-partum weeks, affiliation among adults was negatively associated with urinary OT levels in all individuals. This result is surprising given that OT typically shows a positive rather than negative association with affiliative behaviors (Crockford et al., 2013; Insel, 2010; Snowdon et al., 2010; but see Bartz et al., 2011; De Dreu, 2012; Mustoe et al., 2015). However, this effect may reflect a simple time-budget trade-off and altered social dynamics in groups with new dependent offspring. During early infancy, social interactions are mainly determined by the intense care for infants, leaving less time to engage in affiliation with other 
group members in general. It may be particularly difficult to engage in affiliation with a specific partner since the probability is even smaller that both partners of a specific dyad are available. This is relevant in the face of recent findings that in chimpanzees (Crockford et al., 2013) and marmosets (Finkenwirth et al., 2015), urinary OT responses to adult-adult affiliation are dyadspecific and predominantly occur in strong bond dyads. Furthermore, individuals with high levels of adult-adult affiliation are more likely to engage in infant-care (Finkenwirth and Burkart, in preparation). Together, this thus suggests that the negative effect in our data reflects a lack of affiliative interactions with stronger bonded or preferred group members.

Finally, in the full model, we also found a negative effect of care-taking experience on OT. This effect could tentatively be interpreted as a customization to infant cues, leading to decreasing OT responses in more experienced care-takers. Experience might potentially also complement motivation-stimulating OT effects, in that motivation-stimulation is rather important for less experienced individuals, such as alloparental helpers. However, especially in primiparous mothers, care-motivation should be facilitated and maintained independently of previous alloparenting experience (e.g. via hormonal stimulation), since maternal care is most important during the early post-partum period (Koenig and Rothe, 1991; Mills et al., 2004). This is also evident from experience-independent maternal performance in marmosets (Tardif et al., 1984). It is important to note, however, that we used a combined measure of experience for helpers and breeders, and we cannot exclude the possibility that parenting experience of breeders is qualitatively different from alloparenting experience of non-reproductive helpers. Furthermore, helpers had significantly less experience than breeders (median experience in breeders and helpers was 4 and 1.5 raised infants, respectively; Mann-Whitney $U=23, \mathrm{~N}_{\text {breeders }}=16, \mathrm{~N}_{\text {helpers }}=$ $26, P<0.001, Z=-4.88, r=0.75)$, and we had no inexperienced breeders in the sample. Hence, 
the experience effects reported here should be evaluated with caution, and a potential interaction between motivational and experience-related compensating OT effects requires further investigation.

In this study we chose a non-invasive method of OT sampling. Crockford et al. (2014) review and discuss the relevance of peripheral OT measures for studying the dynamics of social interactions and relationships with particular emphasis on urinary OT. Besides its effects in social bonding and affiliation in mammals (Lim and Young, 2006), peripheral OT is involved in female reproduction (labor and milk letdown: Blanks and Thornton, 2003; Uvnäs-Moberg et al., 2001) and anxiety reduction (Olff et al., 2013). A broadening body of evidence indicates that these central and peripheral OT pathways can be coordinated (Carson et al., 2014; Carter et al., 2007; O'Byrne et al., 1990; Ross and Young, 2009; Wotjak et al., 1998) and may exert crosslinked effects (Ayers et al., 2011; Madden and Clutton-Brock, 2011; Witt et al., 1990). Other studies indicate independent control mechanisms of central and peripheral OT secretion (Amico et al., 1990; Rosenblum et al., 2002; Seckl and Lightman, 1987). However, a coordination of central and peripheral OT effects may also be facilitated indirectly, e.g. via steroid hormones (Bos et al., 2012; McCarthy, 1995; Ochedalski et al., 2007) and bidirectional feedback mechanisms with peripheral organs and body states (Goodson and Thompson, 2010). Such a coordination may also be dependent on the involved stimulating brain regions (MartínezLorenzana et al., 2008). In primates, urinary OT levels have been found to be related to affiliative (Crockford et al., 2013; Finkenwirth et al., 2015; Wittig et al., 2014) and socio-sexual interactions (Moscovice and Ziegler, 2012; Snowdon et al., 2010) among group-living individuals. Accordingly, Crockford et al. (2014) suggested that peripheral OT measurements are indeed useful to capture central OT excretion or a coordinated release between central and 
peripheral effects in primates. However, experimental studies looking at exogenous OT effects on care-taking behaviors and motivation would be valuable to better understand the effects and potential causal mechanisms related to the findings of our study.

\section{Conclusion}

Our findings show that OT is positively associated with care-taking behaviors in groupliving marmoset parents and alloparents. OT increased in all adult group members immediately after the birth of infants. Consistent with the changing needs during infant development, OT was correlated with infant-licking during early infancy and with proactive food sharing especially during late infancy. OT did not simply reflect a physical response to care-taking effort, since there was no link to infant-carrying. Rather, OT may be positively associated with care-taking motivation, which is arguably best quantified by proactive food sharing. Importantly, these effects were not only present in breeders but also in alloparental helpers, which makes these findings valuable for a better understanding of the regulation of alloparental care in marmosets, and perhaps also of other cooperatively breeding species such as humans (Burkart and Finkenwirth, 2014). Future studies will have to integrate these findings with effects of other hormones, such as prolactin, that are also positively involved in the regulation of care-taking behavior and that might potentially interact with and compensate OT effects.

\section{Acknowledgements}

We thank H. Galli, T. Bischof, and A. Kissling for assistance and urine collection in the Primate Station at the University of Zurich. We further thank E. Willems and R. Mundry for statistical support. This study was funded by the Swiss National Science Foundation project 
310030_130383 to J. B. and by the Basler Stiftung für Biologische Forschung to C.F.. The

protocol was approved by the Cantonal Commission for Animal Experiments of Canton Zurich, license nr. 150_210.

\section{References}

Albuquerque, F.S., 1999. Cuidado cooperativo à prole em Callithrix jacchus: Dinâmica em ambiente natural. Universidade de Sao Paulo, Sao Paulo.

Almond, R.E.A., Brown, G.R., Keverne, E.B., 2006. Suppression of prolactin does not reduce infant care by parentally experienced male common marmosets (Callithrix jacchus). Horm Behav 49, 673-680.

Amico, J.A., Challinor, S.M., Cameron, J.L., 1990. Pattern of oxytocin concentrations in the plasma and cerebrospinal fluid of lactating rhesus monkeys (Macaca mulatto): Evidence for functionally independent oxytocinergic pathways in primates. J Clin Endocrinol Metab 71, 1531-1535.

Anzenberger, G., Gossweiler, H., 1993. How to obtain individual urine samples from undisturbed marmoset families. Am J Primatol 31, 223-230.

Ayers, L.W., Missig, G., Schulkin, J., Rosen, J.B., 2011. Oxytocin reduces background anxiety in a fear-potentiated startle paradigm: Peripheral vs central administration. Neuropsychopharmacology 36, 2488-2497.

Bahr, N.I., Martin, R.D., Pryce, C.R., 2001. Peripartum sex steroid profiles and endocrine correlates of postpartum maternal behavior in captive gorillas (Gorilla gorilla gorilla). Horm Behav 40, 533-541.

Bales, K.L., Kim, A.J., Lewis-Reese, A.D., Sue Carter, C., 2004. Both oxytocin and vasopressin may influence alloparental behavior in male prairie voles. Horm Behav 45, 354-361.

Bales, K.L., van Westerhuyzen, J.A., Lewis-Reese, A.D., Grotte, N.D., Lanter, J.A., Carter, C.S., 2007. Oxytocin has dose-dependent developmental effects on pair-bonding and alloparental care in female prairie voles. Horm Behav 52, 274-279.

Bardi, M., French, J.A., Ramirez, S.M., Brent, L., 2004. The role of the endocrine system in baboon maternal behavior. Biol Psychiatry 55, 724-732.

Bardi, M., Shimizu, K., Barrett, G.M., Borgognini-Tarli, S.M., Huffman, M.A., 2003. Peripartum sex steroid changes and maternal style in rhesus and Japanese macaques. Gen Comp Endocrinol 133, 323331.

Bartz, J.A., Zaki, J., Bolger, N., Ochsner, K.N., 2011. Social effects of oxytocin in humans: context and person matter. Trends Cogn Sci 15, 301-309.

Blanks, A.M., Thornton, S., 2003. The role of oxytocin in parturition. BJOG 110, 46-51.

Bos, P.A., Panksepp, J., Bluthé, R.-M., Honk, J.v., 2012. Acute effects of steroid hormones and neuropeptides on human social-emotional behavior: A review of single administration studies. Front Neuroendocrinol 33, 17-35.

Broad, K.D., Curley, J.P., Keverne, E.B., 2006. Mother-infant bonding and the evolution of mammalian social relationships. Phil Trans R Soc B 361, 2199-2214.

Brown, G.R., Almond, R.E.A., Bergen, Y.v., 2004. Begging, stealing, and offering: food transfer in nonhuman primates, Advances in the Study of Behavior. Academic Press, pp. 265-295.

Burkart, J., Finkenwirth, C., 2014. Marmosets as model species in neuroscience and evolutionary anthropology. Neuroscience Research. 
Carson, D.S., Berquist, S.W., Trujillo, T.H., Garner, J.P., Hannah, S.L., Hyde, S.A., Sumiyoshi, R.D., Jackson, L.P., Moss, J.K., Strehlow, M.C., Cheshier, S.H., Partap, S., Hardan, A.Y., Parker, K.J., 2014. Cerebrospinal fluid and plasma oxytocin concentrations are positively correlated and negatively predict anxiety in children. Mol Psychiatry 20, 1085-1090.

Carter, C.S., Pournajafi-Nazarloo, H., Kramer, K.M., Ziegler, T.E., White-Traut, R., Bello, D., Schwertz, D., 2007. Behavioral associations and potential as a salivary biomarker. Ann N Y Acad Sci 1098, 312-322.

Carter, S.C., 1998. Neuroendocrine perspectives on social attachment and love. Psychoneuroendocrinology 23, 779-818.

Chambers, P.L., Hearn, J.P., 1979. Peripheral plasma levels of progesterone, oestradiol-17 $\beta$, oestrone, testosterone, androstenedione and chorionic gonadotrophin during pregnancy in the marmoset monkey, Callithrix jacchus. J Reprod Fertil 56, 23-32.

Crockford, C., Deschner, T., Ziegler, T.E., Wittig, R.M., 2014. Endogenous peripheral oxytocin measures can give insight into the dynamics of social relationships: a review. Front Behav Neurosci 8, 68. Crockford, C., Wittig, R.M., Langergraber, K., Ziegler, T.E., Zuberbühler, K., Deschner, T., 2013. Urinary oxytocin and social bonding in related and unrelated wild chimpanzees. Proc R Soc B 280.

De Dreu, C.K., 2012. Oxytocin modulates cooperation within and competition between groups: an integrative review and research agenda. Horm Behav 61, 419-428.

Digby, L., 1995. Infant care, infanticide, and female reproductive strategies in polygynous groups of common marmosets (Callithrix jacchus). Behav Ecol Sociobiol 37, 51-61.

Digby, L.J., Ferrari, S.F., Saltzman, W., 2007. Callitrichines: the role of competition in cooperatively breeding species., in: Campbell, C.J., Fuentes, A., MacKinnon, K.C., Panger, M.A., Bearder, S.K. (Eds.), Primates in perspective. Oxford University Press, New York, pp. 85-105.

Dixson, A.F., George, L., 1982. Prolactin and parental behaviour in a male New World primate. Nature 299, 551-553.

Fahrbach, S.E., Morrell, J.I., Pfaff, D.W., 1984. Oxytocin induction of short-latency maternal behavior in nulliparous, estrogen-primed female rats. Horm Behav 18, 267-286.

Feldman, R., 2007. Parent-infant synchrony: biological foundations and developmental outcomes. Curr Dir Psychol Sci 16, 340-345.

Feldman, R., Gordon, I., Zagoory-Sharon, O., 2011. Maternal and paternal plasma, salivary, and urinary oxytocin and parent-infant synchrony: considering stress and affiliation components of human bonding. Dev sci 14, 752-761.

Finkenwirth, C., Burkart, J.M., in preparation. Consequences of relationship quality on infantcare behavior in group-living marmosets.

Finkenwirth, C., van Schaik, C., Ziegler, T.E., Burkart, J.M., 2015. Strongly bonded family members in common marmosets show synchronized fluctuations in oxytocin. Physiol Behav 151, 246251.

Fite, J.E., French, J.A., 2000. Pre- and postpartum sex steroids in female marmosets (Callithrix kuhlii): is there a link with infant survivorship and maternal behavior? Horm Behav 38, 1-12.

Fleming, A.S., Steiner, M., Anderson, V., 1987. Hormonal and attitudinal correlates of maternal behaviour during the early postpartum period in first-time mothers. J Reprod Infant Psychol 5, 193-205.

Garber, P.A., 1997. One for all and breeding for one: cooperation and competition as a tamarin reproductive strategy. Evol Anthropol 5, 187-199.

Garber, P.A., Porter, L.M., Spross, J., Fiore, A.D., 2015. Tamarins: Insights into monogamous and non-monogamous single female social and breeding systems. Am J Primatol.

Goldizen, A.W., 1987. Tamarins and marmosets: communal care of offspring., in: Smuts, B.B., Cheney, D.L., Seyfarth, R.M., Wrangham, R.W., Struhsaker, T.T. (Eds.), Primate societies. University of Chicago Press, London, pp. 34-43.

Goldizen, A.W., 1988. Tamarin and marmoset mating systems: Unusual flexibility. Trends in Ecology \& Evolution 3, 36-40. 
Goldizen, A.W., 2003. Social monogamy and its variations in callitrichids: do these relate to the costs of infant care?, in: Reichard, U.H., Boesch, C. (Eds.), Monogamy: mating strategies and partnerships in birds, humans and other mammals. Cambridge University Press, Cambridge, United Kingdom, pp. 232-247.

Goodson, J.L., Thompson, R.R., 2010. Nonapeptide mechanisms of social cognition, behavior and species-specific social systems. Curr Opin Neurobiol 20, 784-794.

Gordon, I., Zagoory-Sharon, O., Leckman, J.F., Feldman, R., 2010. Oxytocin and the development of parenting in humans. Biol Psychiatry 68, 377-382.

Hirst, J.J., Thorburn, G.D., 1996. Initiation of parturition in non-human primates. Adv Organ Biol $1,61-87$.

Insel, T.R., 2010. The challenge of translation in social neuroscience: a review of oxytocin, vasopressin, and affiliative behavior. Neuron 65, 768-779.

Jaeggi, A.V., Gurven, M., 2013. Natural cooperators: food sharing in humans and other primates. Evol Anthropol 22, 186-195.

Kendrick, K.M., 2000. Oxytocin, motherhood and bonding. Exp Physiol 85, 111-124.

Kendrick, K.M., Keverne, E.B., 1989. Release of monoamines, amino acids and oxytocin from the sheep brain during parturition and suckling. Curr Sep 9, 114.

Kendrick, K.M., Keverne, E.B., Baldwin, B.A., 1987. Intracerebroventricular oxytocin stimulates maternal behaviour in the sheep. Neuroendocrinology 46, 56-61.

Kholkute, S.D., 1984. Plasma estradiol and progesterone levels during post-partum ovulation and early pregnancy in the common marmoset, Callithrix jacchus. Primates 25, 538-543.

Koenig, A., 1995. Group size, composition, and reproductive success in wild common marmosets (Callithrix jacchus). Am J Primatol 35, 311-317.

Koenig, A., Rothe, H., 1991. Social relationships and individual contribution to cooperative behaviour in captive common marmosets (Callithrix jacchus). Primates 32, 183-195.

Landgraf, R., Schulz, J.G., Eulenberger, K., Wilhelm, J., 1983. Plasma levels of oxytocin and vasopressin before, during and after parturition in cows. Exp Clin Endocrinol 81, 321-328.

Lim, M.M., Young, L.J., 2006. Neuropeptidergic regulation of affiliative behavior and social bonding in animals. Horm Behav 50, 506-517.

Madden, J.R., Clutton-Brock, T.H., 2011. Experimental peripheral administration of oxytocin elevates a suite of cooperative behaviours in a wild social mammal. Proc R Soc B 278, 1189-1194.

Maestripieri, D., 2001a. Biological bases of maternal attachment. Curr Dir Psychol Sci 10, 79-83.

Maestripieri, D., 2001b. Is there mother-infant bonding in primates? Dev Rev 21, 93-120.

Martínez-Lorenzana, G., Espinosa-López, L., Carranza, M., Aramburo, C., Paz-Tres, C., RojasPiloni, G., Condés-Lara, M., 2008. PVN electrical stimulation prolongs withdrawal latencies and releases oxytocin in cerebrospinal fluid, plasma, and spinal cord tissue in intact and neuropathic rats. Pain 140, 265-273.

Martins, E., Finkenwirth, C., Burkart, J., in preparation. Food sharing patterns in marmoset monkeys. To each according to its need?

McCarthy, M., 1995. Estrogen modulation of oxytocin and its relation to behavior. Adv Exp Med Biol 395, 235-245.

McNeilly, A.S., Abbott, D.H., Lunn, S.F., Chambers, P.C., Hearn, J.P., 1981. Plasma prolactin concentrations during the ovarian cycle and lactation and their relationship to return of fertility post partum in the common marmoset (Callithrix jacchus). J Reprod Fertil 62, 353-360.

Mills, D.A., Windle, C.P., Baker, H.F., Ridley, R.M., 2004. Analysis of infant-carrying in large, well-established family groups of captive marmosets (Callithrix jacchus). Primates 45, 259-265.

Morris, M., Stevens, S.W., Adams, M.R., 1980. Plasma oxytocin during pregnancy and lactation in the cynomolgus monkey. Biol Reprod 23, 782-787.

Moscovice, L.R., Ziegler, T.E., 2012. Peripheral oxytocin in female chacma baboons relates to estrus and maintenance of sexual consortships. Horm Behav 62, 592-597. 
Mota, M.T.d.S., Franci, C.R., de Sousa, M.B.C., 2006. Hormonal changes related to paternal and alloparental care in common marmosets (Callithrix jacchus). Horm Behav 49, 293-302.

Mota, M.T.d.S., Sousa, M.B.C., 2000. Prolactin levels of fathers and helpers related to alloparental care in common marmosets, Callithrix jacchus. Folia Primatol 71, 22-26.

Mustoe, A.C., Cavanaugh, J., Harnisch, A.M., Thompson, B.E., French, J.A., 2015. Do marmosets care to share? Oxytocin treatment reduces prosocial behavior toward strangers. Horm Behav 71, 83-90.

Nissen, E., Lilja, G., Widström, A.-M., Uvnás-Moberg, K., 1995. Elevation of oxytocin levels early post-partum in women. Acta Obstet Gynecol Scand 74, 530-533.

Novy, M.J., Haluska, G.J., 1994. New perspectives on estrogen, progesterone, and oxytocin action in primate parturition, in: Chwalisz, K., Garfield, R.E. (Eds.), Basic mechanisms controlling term and preterm birth. Springer Berlin Heidelberg, pp. 163-195.

O'Byrne, K.T., Lunn, S.F., Coen, C.W., 1990. Central oxytocin stimulates luteinizing hormone release in the marmoset, a primate which fails to show lactationally-induced infertility. J Neuroendocrinol $2,419-421$.

Ochedalski, T., Subburaju, S., Wynn, P.C., Aguilera, G., 2007. Interaction between oestrogen and oxytocin on hypothalamic-pituitary-adrenal axis activity. J Neuroendocrinol 19, 189-197.

Olff, M., Frijling, J.L., Kubzansky, L.D., Bradley, B., Ellenbogen, M.A., Cardoso, C., Bartz, J.A., Yee, J.R., van Zuiden, M., 2013. The role of oxytocin in social bonding, stress regulation and mental health: An update on the moderating effects of context and interindividual differences. Psychoneuroendocrinology 38, 1883-1894.

Pedersen, C.A., Caldwell, J.D., Walker, C., Ayers, G., Mason, G.A., 1994. Oxytocin activates the postpartum onset of rat maternal behavior in the ventral tegmental and medial preoptic areas. Behav Neurosci 108, 1163-1171.

Price, E.C., 1991. Competition to carry infants in captive families of cotton-top tamarins (Saguinus Oedipus). Behav 118, 66-88.

Pryce, C.R., 1993. The regulation of maternal behaviour in marmosets and tamarins. Behav Process 30, 201-224.

Pryce, C.R., Abbott, D.H., Hodges, J.K., Martin, R.D., 1988. Maternal behavior is related to prepartum urinary estradiol levels in red-bellied tamarin monkeys. Physiol Behav 44, 717-726.

Pryce, C.R., Döbeli, M., Martin, R.D., 1993. Effects of sex steroids on maternal motivation in the common marmoset (Callithrix jacchus): Development and application of an operant system with maternal reinforcement. J Comp Psychol 107, 99-115.

Pryce, C.R., Mutschler, T., Döbeli, M., Nievergelt, C., Martin, R.D., 1995. Prepartum sex steroid hormones and infant-directed behaviour in primiparous marmoset mothers (Callithrix jacchus), in: Pryce, C.R., Martin, R.D., Skuse, D. (Eds.), Motherhood in human and nonhuman primates: Biosocial determinants, 3rd Schultz-Biegert Symposium, Kartause Ittingen. Karger, Basel, pp. 78-86.

Roberts, R.L., Jenkins, K.T., Lawler, J.T., Wegner, F.H., Norcross, J.L., Bernhards, D.E., Newman, J.D., 2001. Prolactin levels are elevated after infan-009 carrying in parentally inexperienced common marmosets. Physiol Behav 72, 713-720.

Rosenblum, L.A., Smith, E., Altemus, M., Scharf, B.A., Owens, M.J., Nemeroff, C.B., Gorman, J.M., Coplan, J.D., 2002. Differing concentrations of corticotropin-releasing factor and oxytocin in the cerebrospinal fluid of bonnet and pigtail macaques. Psychoneuroendocrinology 27, 651-660.

Ross, H.E., Young, L.J., 2009. Oxytocin and the neural mechanisms regulating social cognition and affiliative behavior. Front Neuroendocrinol 30, 534-547.

Rothe, H., Koenig, A., Darms, K., 1993. Infant survival and number of helpers in captive groups of common marmosets (Callithrix jacchus). Am J Primatol 30, 131-137.

Saito, A., Nakamura, K., 2011. Oxytocin changes primate paternal tolerance to offspring in food transfer. J Comp Physiol A 197, 329-337.

Saltzman, W., Abbott, D.H., 2005. Diminished maternal responsiveness during pregnancy in multiparous female common marmosets. Horm Behav 47, 151-163. 
Saltzman, W., Maestripieri, D., 2011. The neuroendocrinology of primate maternal behavior. Prog Neuropsychopharmacol Biol Psychiatry 35, 1192-1204.

Schradin, C., Anzenberger, G., 2001. Costs of infant carrying in common marmosets, Callithrix jacchus: an experimental analysis. Anim Behav 62, 289-295.

Schradin, C., Anzenberger, G., 2004. Development of prolactin levels in marmoset males: From adult son to first-time father. Horm Behav 46, 670-677.

Schradin, C., Reeder, D.M.; Mendoza, S.P.; Anzenberger, G., 2003. Prolactin and paternal care: Comparison of three species of monogamous new world monkeys (Callicebus cupreus, Callithrix jacchus, and Callimico goeldii). J Comp Psychol, Vol 117(2), Jun 2003, 166-175.

Seckl, J.R., Lightman, S.L., 1987. Diurnal rhythm of vasopressin but not of oxytocin in the cerebrospinal fluid of the goat: Lack of association with plasma cortisol rhythm. J Endocrinol 114, 477482.

Seltzer, L.J., Ziegler, T.E., 2007. Non-invasive measurement of small peptides in the common marmoset (Callithrix jacchus): a radiolabeled clearance study and endogenous excretion under varying social conditions. Horm Behav 51, 436-442.

Snowdon, C.T., Cronin, K.A., 2007. Cooperative breeders do cooperate. Behav Process 76, 138.

Snowdon, C.T., Pieper, B.A., Boe, C.Y., Cronin, K.A., Kurian, A.V., Ziegler, T.E., 2010. Variation in oxytocin is related to variation in affiliative behavior in monogamous, pairbonded tamarins. Horm Behav 58, 614-618.

Solomon, N., French, J.A., 1997. Cooperative breeding in mammals. Cambridge University Press, New York.

Sussman, R.W., Garber, P.A., 1987. A new interpretation of the social organization and mating system of the callitrichidae. Int J Primatol 8, 73-92.

Tardif, S.D., Richter, C.B., Carson, R.L., 1984. Effects of sibling-rearing experience on future reproductive success in two species of callitrichidae. Am J Primatol 6, 377-380.

Uvnäs-Moberg, K., 1996. Neuroendocrinology of the mother-child interaction. Trends Endocrinol Metab 7, 126-131.

Uvnäs-Moberg, K., Johansson, B., Lupoli, B., Svennersten-Sjaunja, K., 2001. Oxytocin facilitates behavioural, metabolic and physiological adaptations during lactation. Appl Anim Behav Sci 72, 225234.

Witt, D.M., Carter, C.S., Walton, D.M., 1990. Central and peripheral effects of oxytocin administration in prairie voles (Microtus ochrogaster). Pharmacol Biochem Behav 37, 63-69.

Wittig, R.M., Crockford, C., Deschner, T., Langergraber, K.E., Ziegler, T.E., Zuberbühler, K., 2014. Food sharing is linked to urinary oxytocin levels and bonding in related and unrelated wild chimpanzees. Proc R Soc B 281.

Wotjak, C.T., Ganster, J., Kohl, G., Holsboer, F., Landgraf, R., Engelmann, M., 1998. Dissociated central and peripheral release of vasopressin, but not oxytocin, in response to repeated swim stress: New insights into the secretory capacities of peptidergic neurons. Neuroscience 85, 1209-1222.

Yamamoto, M.E., Araujo, A., Arruda, M.d.F., Lima, A.K.M., Siqueira, J.d.O., Hattori, W.T., 2014. Male and female breeding strategies in a cooperative primate. Behav Process 109, Part A, 27-33.

Yamamoto, M.E., Araújo, A., de Sousa, M.B.C., Arruda, M.d.F., 2010. Chapter 8 - Social Organization in Callithrix jacchus: Cooperation and Competition, in: Regina, M. (Ed.), Advances in the Study of Behavior. Academic Press, pp. 259-273.

Yamamoto, M.E., Arruda, M.F., Alencar, A.I., Sousa, M.B.C., Araújo, A., 2009. Mating systems and female-female competition in the common marmoset, Callithrix jacchus, in: Ford, S.M., Porter, L.M., Davis, L.C. (Eds.), The Smallest Anthropoids. Springer US, pp. 119-133.

Yamamoto, M.E., Box, H.O., 1997. The role of non-reproductive helpers in infant care in captive Callithrix jacchus. Ethol 103, 760-771.

Zahed, S.R., Kurian, A.V., Snowdon, C.T., 2010. Social dynamics and individual plasticity of infant care behavior in cooperatively breeding cotton-top tamarins. Am J Primatol 72, 296-306. 
Ziegler, T.E., Snowdon, C.T., 2000. Preparental hormone levels and parenting experience in male cotton-top tamarins, Saguinus oedipus. Horm Behav 38, 159-167.

Ziegler, T.E., Washabaugh, K.F., Snowdon, C.T., 2004. Responsiveness of expectant male cotton-top tamarins, Saguinus oedipus, to mate's pregnancy. Horm Behav 45, 84-92.

Ziegler, T.E., Wegner, F.H., Snowdon, C.T., 1996. Hormonal responses to parental and nonparental conditions in male cotton-top tamarins, Saguinus oedipus, a new world primate. Horm Behav 30, 287-297. 


\section{Tables}

Table 1: Linear mixed-effects model fit by REML on urinary oxytocin, comparing late prepartum and early post-partum periods (model 1). Parameter estimates: factor levels with bars are compared to the remaining factor levels. Bold: $\mathrm{p}<0.05$. Late pre-partum period: week -1 ; early post-partum period: week 0-1 ( $\mathrm{N}=70$ samples from 26 individuals in 8 reproductive cycles).

\begin{tabular}{lccllllll}
\hline \hline \multicolumn{1}{c}{ fixed factor } & $\mathbf{F}$ & df & p-value & factor level & estimate & s.e. & t & P \\
\hline \hline intercept & 0.04 & 1 & 0.842 & & -0.75 & 0.39 & -1.92 & 0.062 \\
\hline $\begin{array}{l}\text { sampling } \\
\text { period }\end{array}$ & $\mathbf{2 6 . 0 6}$ & $\mathbf{1}$ & $<\mathbf{0 . 0 0 0 1}$ & post-partum & $\mathbf{0 . 9 6}$ & $\mathbf{0 . 1 9}$ & $\mathbf{4 . 9 8}$ & $<\mathbf{0 . 0 0 0 1}$ \\
status & & & & pre-partum & - & - & - & - \\
& $\mathbf{0 . 1 5}$ & $\mathbf{1}$ & $\mathbf{0 . 7 0 7}$ & helper & $\mathbf{- 0 . 7 9}$ & $\mathbf{0 . 2 7}$ & $\mathbf{- 2 . 9 3}$ & $\mathbf{0 . 0 0 9}$ \\
sex & & & & breeder & - & - & - & - \\
& $\mathbf{6 . 3 2}$ & $\mathbf{1}$ & $\mathbf{0 . 0 2 2}$ & male & $\mathbf{- 1 . 3 2}$ & $\mathbf{0 . 3 0}$ & $\mathbf{- 4 . 4 1}$ & $<\mathbf{0 . 0 0 1}$ \\
& & & & female & - & - & - & - \\
status*sex & $\mathbf{1 3 . 2 0}$ & $\mathbf{1}$ & $\mathbf{0 . 0 0 2}$ & & $\mathbf{1 . 4 0}$ & $\mathbf{0 . 3 8}$ & $\mathbf{3 . 6 3}$ & $\mathbf{0 . 0 0 2}$ \\
\hline \hline
\end{tabular}

Table 2: Linear mixed-effects model fits by REML on urinary oxytocin during early and

late infancy. Parameter estimates: factor levels with bars are compared with remaining factor levels. Bold: $\mathrm{p}<0.05$. Early infancy: post-partum week 0-5 (model 7); late infancy: post-partum week 6-11 (model 8). ( $\mathrm{N}=236$ samples from 22 individuals in 5 reproductive cycles).

\begin{tabular}{|c|c|c|c|c|c|c|c|c|c|}
\hline model & fixed factor & $\mathbf{F}$ & df & p-value & factor level & estimate & s.e. & $\mathbf{t}$ & $\mathbf{P}$ \\
\hline \multirow{9}{*}{$\begin{array}{l}\text { model } \\
7 \text {, early } \\
\text { infancy }\end{array}$} & intercept & 0.38 & 1 & 0.538 & & 0.94 & 0.41 & 2.28 & 0.025 \\
\hline & status & 0.45 & 1 & 0.513 & helper & -0.85 & 0.34 & -2.48 & 0.026 \\
\hline & & & & & breeder & - & - & - & - \\
\hline & sex & 1.79 & 1 & 0.200 & male & -0.96 & 0.33 & -2.93 & 0.010 \\
\hline & & & & & female & - & - & - & - \\
\hline & status*sex & 4.21 & 1 & 0.058 & & 0.91 & 0.45 & 2.05 & 0.058 \\
\hline & $\begin{array}{l}\text { adult- } \\
\text { affiliation }\end{array}$ & 18.18 & 1 & $<0.0001$ & & -0.28 & 0.07 & -4.10 & 0.0001 \\
\hline & $\begin{array}{l}\text { proactive food } \\
\text { sharing }\end{array}$ & 2.17 & 1 & 0.144 & & 1.65 & 1.04 & 1.59 & 0.115 \\
\hline & infant-licking & 7.31 & 1 & 0.008 & & 0.22 & 0.08 & 2.89 & 0.005 \\
\hline
\end{tabular}




\begin{tabular}{|c|c|c|c|c|c|c|c|c|c|}
\hline & $\begin{array}{l}\text { infant- } \\
\text { grooming }\end{array}$ & 0.13 & 1 & 0.720 & & -0.10 & 0.13 & -0.73 & 0.469 \\
\hline & infant-carrying & 0.14 & 1 & 0.708 & & -0.07 & 0.08 & -0.80 & 0.424 \\
\hline \multirow{13}{*}{$\begin{array}{l}\text { model } \\
8, \text { late } \\
\text { infancy }\end{array}$} & intercept & 0.00 & 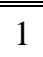 & 0.944 & & 1.07 & 0.69 & 1.55 & 0.126 \\
\hline & \multirow[t]{2}{*}{ status } & 0.13 & 1 & 0.722 & helper & -1.18 & 0.54 & -2.17 & 0.048 \\
\hline & & & & & breeder & - & - & - & - \\
\hline & \multirow[t]{2}{*}{$\operatorname{sex}$} & 0.09 & 1 & 0.771 & male & -0.58 & 0.50 & -1.15 & 0.272 \\
\hline & & & & & female & - & - & - & - \\
\hline & status*sex & 4.62 & 1 & 0.050 & & 1.09 & 0.63 & 1.74 & 0.103 \\
\hline & $\begin{array}{l}\text { care-taking } \\
\text { experience }\end{array}$ & 5.30 & 1 & 0.024 & & -0.08 & 0.08 & -0.99 & 0.328 \\
\hline & $\begin{array}{l}\text { adult- } \\
\text { affiliation }\end{array}$ & 1.31 & 1 & 0.256 & & -0.090 & 0.12 & -0.76 & 0.452 \\
\hline & $\begin{array}{l}\text { proactive } \\
\text { food sharing }\end{array}$ & 0.96 & 1 & 0.330 & & 1.82 & 0.81 & 2.24 & 0.028 \\
\hline & infant-licking & 0.13 & 1 & 0.719 & & -0.08 & 0.16 & 0.51 & 0.615 \\
\hline & $\begin{array}{l}\text { infant- } \\
\text { grooming }\end{array}$ & 0.16 & 1 & 0.690 & & -0.15 & 0.21 & -0.70 & 0.484 \\
\hline & infant-carrying & 1.40 & 1 & 0.240 & & -0.16 & 0.11 & -1.43 & 0.156 \\
\hline & $\begin{array}{l}\text { proactive food } \\
\text { sharing*care- } \\
\text { taking } \\
\text { experience }\end{array}$ & 2.84 & 1 & 0.096 & & -0.31 & 0.19 & -1.68 & 0.096 \\
\hline
\end{tabular}




\section{Figure captions}

Figure 1: Mean urinary oxytocin levels ( $\mathrm{Z} \ln$ pg OT/mg Crt) in the pre- (dashed bars) and postpartum (filled bars) period, comparing breeder and helper females and males. Oxytocin levels in breeder females are significantly higher than in all other status-sex classes, and the post-partum oxytocin increase was significant in all classes of individuals (see model 1, table 1). Border and middle lines of the boxes, the boxes themselves, and horizontal error bars refer to quartiles, median, $50 \%$ of scores, and the smallest and largest scores, respectively.

Figure 2: Longitudinal changes of urinary oxytocin levels over 12 weeks around birth (vertical line), comparing female breeders (black solid line), male breeders (grey solid line), female helpers (black dotted line), and male helpers (grey dotted line). A major increase of oxytocin occurs around infant-birth in all classes of animals. The arrow indicates the onset of infantweaning and solid food ingestion, which coincides with the major drop in breeder female OT levels.

Figure 3: Longitudinal changes of infant-carrying (A), -licking (B), -grooming (C), and mean proactive food sharing (D), split by breeder females (black solid lines), breeder males (grey solid lines), helper females (black dotted lines), and helper males (grey dotted lines). 


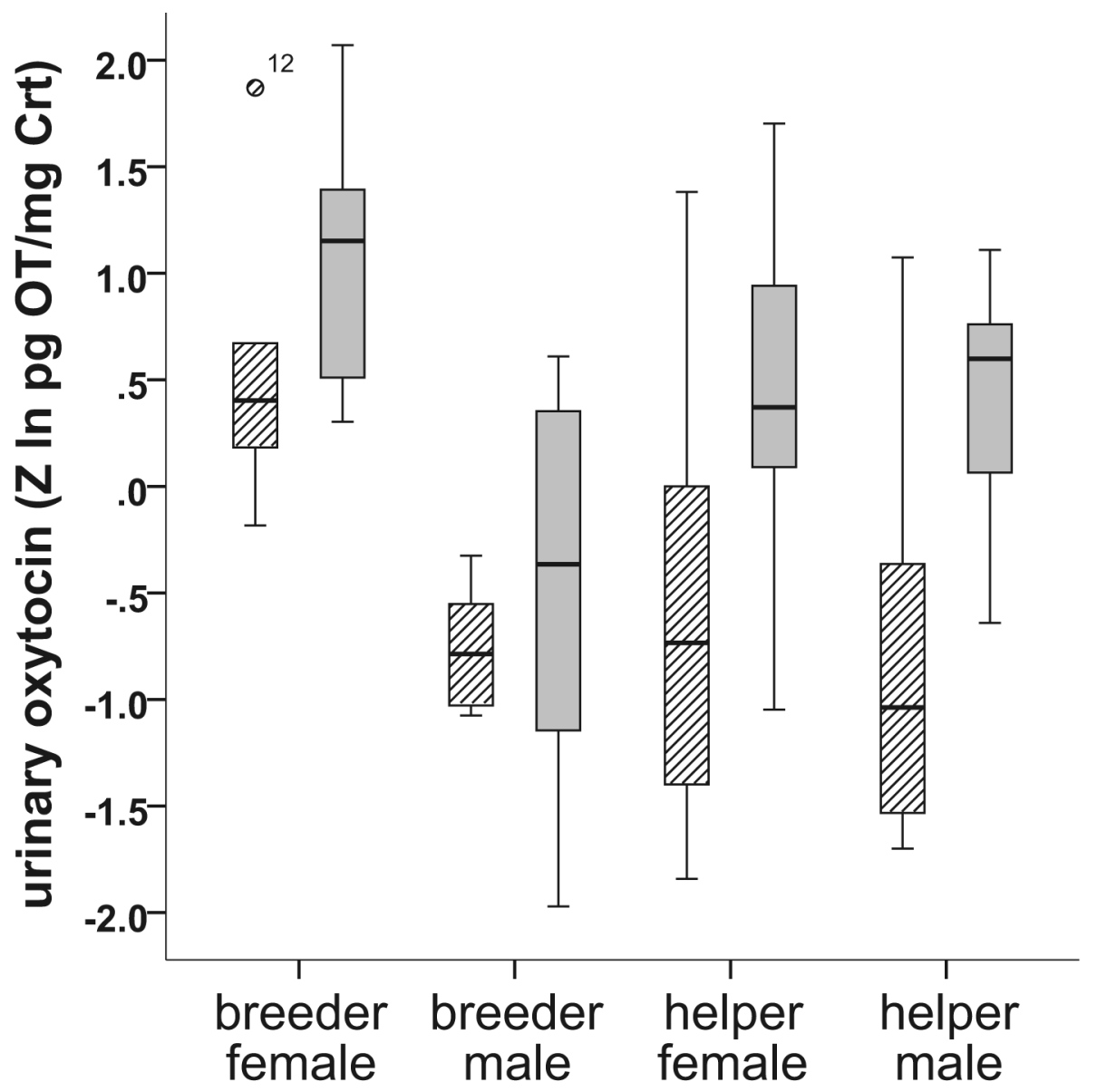




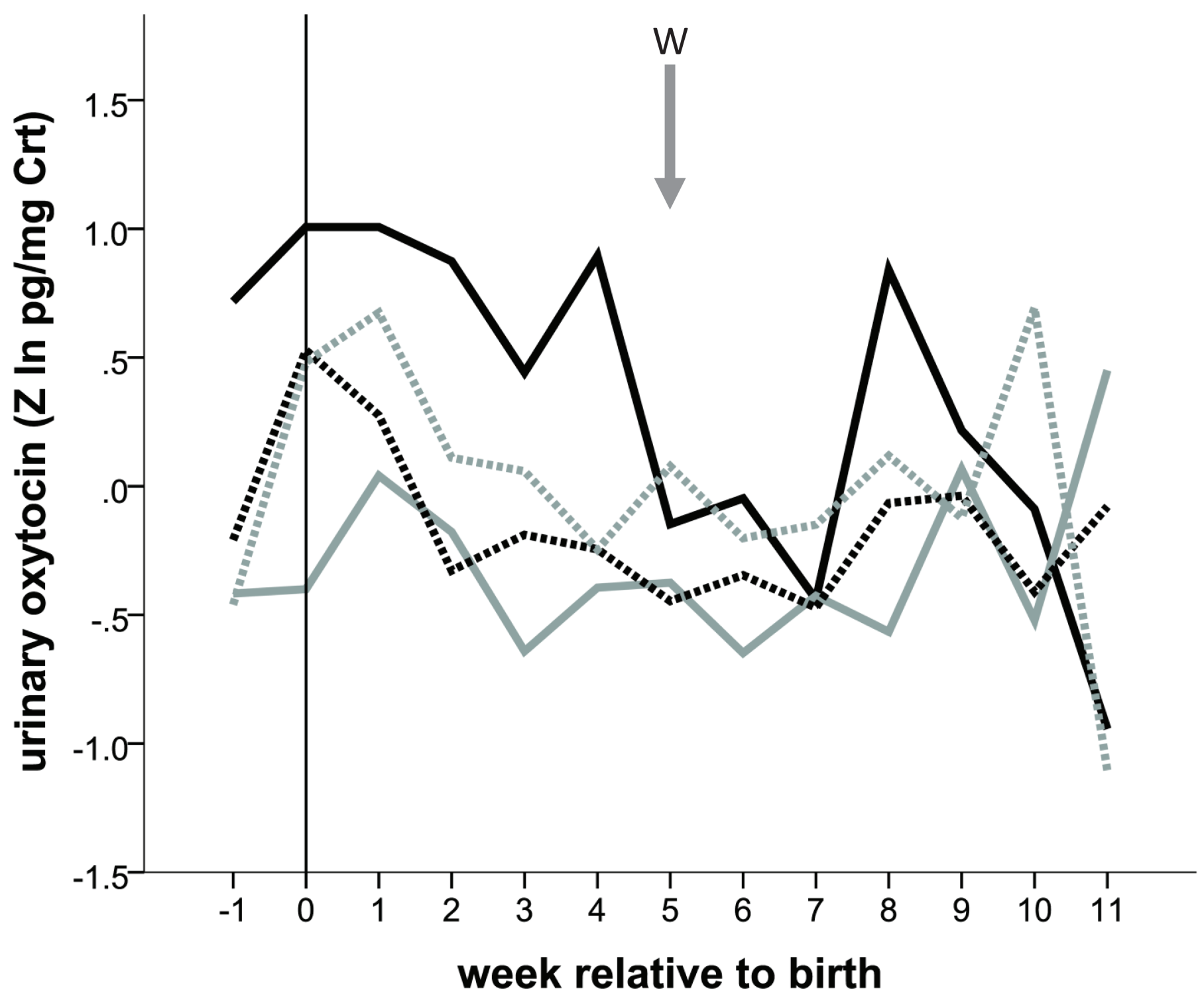



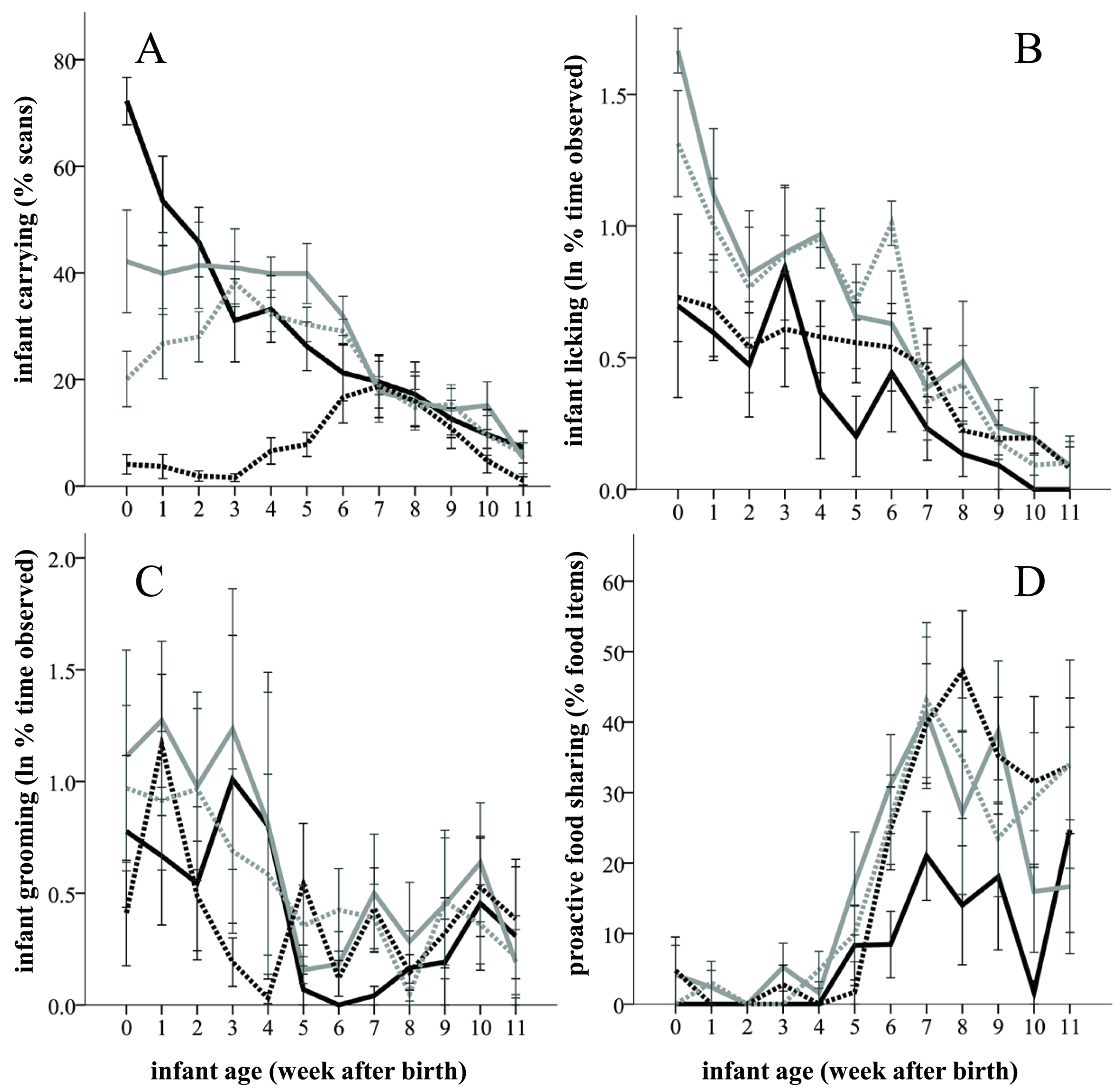